\title{
WILEY-VCH
}

DOI: 10.1002/ ((please add manuscript number))

Article type: Progress Report

\section{Mesoporous matrices as hosts for metal halide perovskite nanocrystals}

Andrea Rubino, ${ }^{1}$ Laura Caliò,${ }^{1}$ Alfonso García-Bennett, ${ }^{2}$ Mauricio E. Calvo, ${ }^{1,}{ }^{*}$ and Hernán Míguez ${ }^{1, *}$

1 Institute of Materials Science of Seville, Spanish National Research CouncilUniversity of Seville, C/Américo Vespucio 49, 41092, Seville, Spain.

2 Department of Molecular Sciences, 4 Wally's Walk, Macquarie University, NSW 2109, Australia.

\section{Abstract}

Several works have recently demonstrated that perovskite nanocrystals can be controllably formed within a variety of porous matrices employing diverse synthetic strategies. By means of the fine tuning of the pore size distribution, the thickness and composition of the walls, the geometry of the void network and its topology, strict control over the structural and morphological parameters of the hosted semiconductor can be achieved, determining its optical absorption and emission properties. Furthermore, porous hosts provide the guest semiconductor with enhanced stability and versatility in terms of processing, which favors its integration in devices. In this Progress Report we present a comprehensive review of the different approaches proposed, as well as a discussion on the relevance they may have for the development of nanostructured perovskite-based optoelectronics. A critical assessment of the optical quality of the hybrid perovskite nanomaterials so obtained, an analysis of the fundamental and applied aspects of the nanocrystal-matrix interaction, and a projected 
prospective of their impact in the fields of artificial lighting and renewable energy is provided.

\section{Introduction}

The use of $\mathrm{ABX}_{3}$ perovskite materials for optoelectronic devices ${ }^{[1-4]}$ has motivated the exploration of a plethora of synthetic approaches with the aim of improving their structural quality ${ }^{[5]}$ and controlling with greater precision their electrical and optical properties. ${ }^{[6]}$ One of the means by which this is achieved is through the reduction of the semiconductor size and dimensionality, ${ }^{[7,8]}$ approaches that have already been put into practice into actual perovskite devices with improved photoluminescence quantum efficiency, spectral properties and stability. ${ }^{[9-14]}$

In this context, a series of recent proposals have made use of porous matrices to achieve a fine control over the semiconductor crystal size at the nanoscale. ${ }^{[15-18]}$ These methods arise as a promising alternative to those based on colloidal nucleation and growth $^{[19-26]}$ for different reasons. First, they do not rely on the use of organic capping layers to prevent the aggregation of the nanocrystals, hence favoring connectivity and electrical transport. ${ }^{[27]}$ At the same time, they might provide a controllable environment to mitigate the different instabilities that affect the performance of these materials. In addition, synthesis within porous templates does not require any additional steps of purification or deposition since, in most cases, the nanocrystals grow directly onto the scaffold walls. When it comes to processing, porous matrices present a large versatility, as they can be attained with a diversity of shapes (nanoparticles, microparticles, thin films, bulk materials) and compositions (metal oxides, polymers). Simultaneously, they allow strict control of the pore size distribution (monomodal, multimodal), morphology, fill factor and topology of the pore network. ${ }^{[28,29]}$ All these features introduce a whole 


\section{WILEY-VCH}

new set of parameters to tune and improve the properties and integration of perovskite nanocrystals in different kinds of device architectures.

This Progress Report is organized as follows. We will first present a description of the routes of impregnation of porous matrices that have been employed with guest perovskite nanocrystals. Then, the different types of porous matrices suitable to host perovskite nanocrystals of controlled size and shape are shown, and their main properties discussed, with focus on the correlation between the characteristics of the matrix and the final structural features of the nanocrystals confined within. An intendedly exhaustive list of all the different attempts to either form or infiltrate either organic or purely inorganic metal halide nanocrystals in a variety of hosts is offered to the reader. Then, an analysis of the optoelectronic properties deployed by the different collections of hosted nanocrystals will be provided, with particular emphasis on light harvesting and emission. Finally, the prospective device integration of materials attained following the templating approach central to this report will be critically discussed.

\section{General characteristics of perovskite impregnation of porous materials}

Independently of the matrix employed, we can distinguish three different methods for the infiltration of $\mathrm{ABX}_{3}$ perovskite nanocrystals ${ }^{[30]}$ within its open pores. These are schematically depicted in Figure 1(a). The first one is a two-step process (labeled as Route I), in which the dissolved metal halide is infiltrated and afterwards the whole scaffold is immersed in a solution containing the A cation (for example methylammonium, MA), so that it penetrates the matrix and reacts with the halide to form the perovskite as the solvent evaporates from the pores. Another possible synthetic route consists in a single step (Route II) where the porous system is impregnated with an 


\section{WILEY-VCH}

unreacted solution of mixed perovskite precursors. Then, the solvent evaporates and the perovskite crystallizes inside the pores after an annealing process. In a general way, for Routes I and II, dispersion of precursors in a porous matrix facilitates nucleation and hinders growth, favoring the formation of nanocrystals. The former is promoted by the presence of walls, whose surface functions as nucleation point, while the latter is reduced by the hampered diffusion of species throughout the network. Thirdly, ex-situ processes (Route III), in which a suspension containing preformed nanocrystals is infiltrated within the porous matrices, have also been developed. In this case, nanocrystal size is predetermined by the colloidal synthesis parameters, and its optical properties and functionalities will only be modified by the interaction with the matrix, but not fully determined by it.

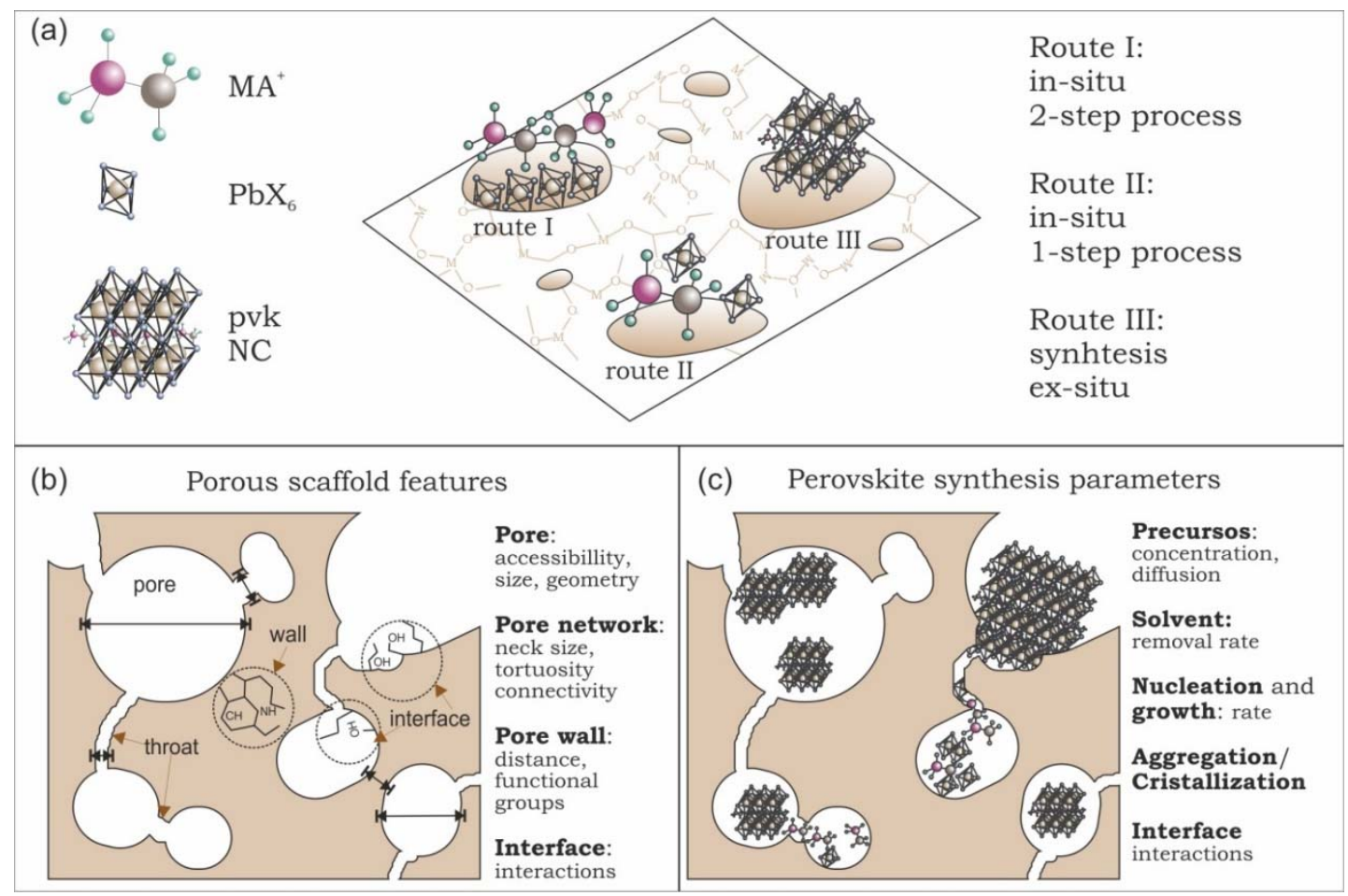

Figure 1. (a) Representative scheme of the different synthetic approaches involving porous materials and $\mathrm{ABX}_{3}$ perovskites (where $\mathrm{MA}=$ methylammonium; $\mathrm{PbX}_{6}=$ lead halide; pvk $\mathrm{NC}=$ perovskite nanocrystal). (b) Scheme of the most important features of pore networks to be considered in the $\mathrm{ABX}_{3}$ synthesis. (c) Controllable parameters for a complete description of the synthesis of $\mathrm{ABX}_{3}$ templated perovskites. 


\section{WILEY-VCH}

When it comes to choose a specific porous scaffold to be used for perovskite nanocrystal impregnation, several factors, schematized in Figure 1(b), should be taken into account. In order to attain a homogeneous dispersion of nanocrystals within the porous scaffold using either precursors (Routes $I$ and II) or pre-synthesized nanocrystals (Route III), the relevant parameters are accessibility (open versus close pores), the size of the pores, the interconnection between them (neck size, number of first neighbors), and tortuosity of the interstitial network. These will determine the dynamics of the chemical reaction taking place within the pores, as the diffusion of the infiltrated species depends on these parameters. For Routes I and II, note that the porous matrix serves as an array of interconnected nano-reactors in which the precursor dispersion is finely distributed. As the volume, and hence the amount of precursor species that will participate in the reaction, is partially restricted by the cage size, formation of crystals of very small size is favored. The tortuosity of the interstitial lattice plays a key role in this latter process. Please notice that for the case of very small pores their geometry could also influence that of the nanocrystals or aggregates of nanocrystals formed inside. ${ }^{[15,18]}$ In the case of Route III, the requirements of good interconnectivity and diffusion become even more crucial, as the pre-formed nanocrystals must flow throughout the host network to uniformly infiltrate it. In this case, pore sizes and necks significantly larger than the average nanocrystal size in the precursor suspension, as well as low tortuosity, are needed in order to achieve proper distribution of crystallites in the host. In this regard, tubular mesostructures of large pore size, which show null interconnectivity with very low tortuosity, are usually preferred over more intricate three dimensional networks to infiltrate preformed nanocrystals. ${ }^{[31]}$

Simultaneously, the interplay between the matrix and the specific synthesis parameters, schematized in Figure 1(c), should also be considered. A high concentration 


\section{WILEY-VCH}

could cause the formation of macrocrystals (Routes I and II) or deposition of aggregates (Route III) at the surface entrance, blocking the access to the inner pores, while low concentration, conversely, may give rise to an insufficient amount of active material and hence to a poor performance of the film as light absorbing or emitting layer. Likewise, the diffusion of species within the pores will also depend strongly on the compatibility between the solvents employed and the matrix. Solvents typically used for the synthesis of perovskites (N,N-Dimethylformamide, Dimethyl sulfoxide, gamma-Butyrolactone, etc.), are aprotic polar solvents, which can present a very different degree of wettability depending on the presence of one type of functional group or another on the pore walls. In I and II routes capillarity plays a central role in the diffusion since the interfacial surface tension between the nanochannel walls (made of, principally, $\mathrm{SiO}_{2}$ or $\mathrm{TiO}_{2}$ ) and most solvents used to disperse the perovskite precursors and infiltrate the porous structures is low. This eases either the diffusion or convection of reagents through the pore network, which leads to a homogeneous infiltration of the matrix with crystallites with a narrow size distribution. However, below a certain threshold pore diameter, nanofluidics phenomena may increase the fluid viscosity and hinder mass transport. ${ }^{[32]}$ This issue can be solved in thin films by forcing the entrance of liquid precursors into the nanochannels by convection (e.g., by spin coating). From another perspective, in the approach we are discussing herein, the growth dynamics and hence the size of the nanocrystals will, to a good extent, be determined by the evaporation rate of the solvent. This implies a dependence on temperature but also on the specific characteristics of the pore network. For instance, an open-pore gyroidal network like KIT-6 provides not only accessible voids to fill with reagents but also will favor the evaporation of the solvent.

\section{Porous matrices as hosts for perovskite nanocrystals}




\section{WILEY-VCH}

In this section we revise the porous matrices that have been used to create confined perovskite nanocrystals. From the early stages of the field, perovskites have been synthesized within unstructured porous matrices of different composition. These porous scaffolds, typically obtained by disorderly packing polydisperse metal oxide particles $\left(\mathrm{TiO}_{2}, \mathrm{SiO}_{2}, \mathrm{Al}_{2} \mathrm{O}_{3}, \mathrm{ZnO}\right)$, have been used to host and support the semiconductor in one of the standard configurations of the solution processed photovoltaic device known as mesoporous perovskite solar cell. More recently, dispersion in polymeric and glassy matrices have been shown to provide perovskite crystallites with enhanced stability ${ }^{[33,34]}$ as well as high photoluminescence quantum yield (PLQY). ${ }^{[35,36]}$ However, it has been only recently that attention has been paid to the opportunities offered by matrices in which a precise control over the pore network can be achieved. In a series of recent papers, different methods have demonstrated that it is possible to synthesize perovskite

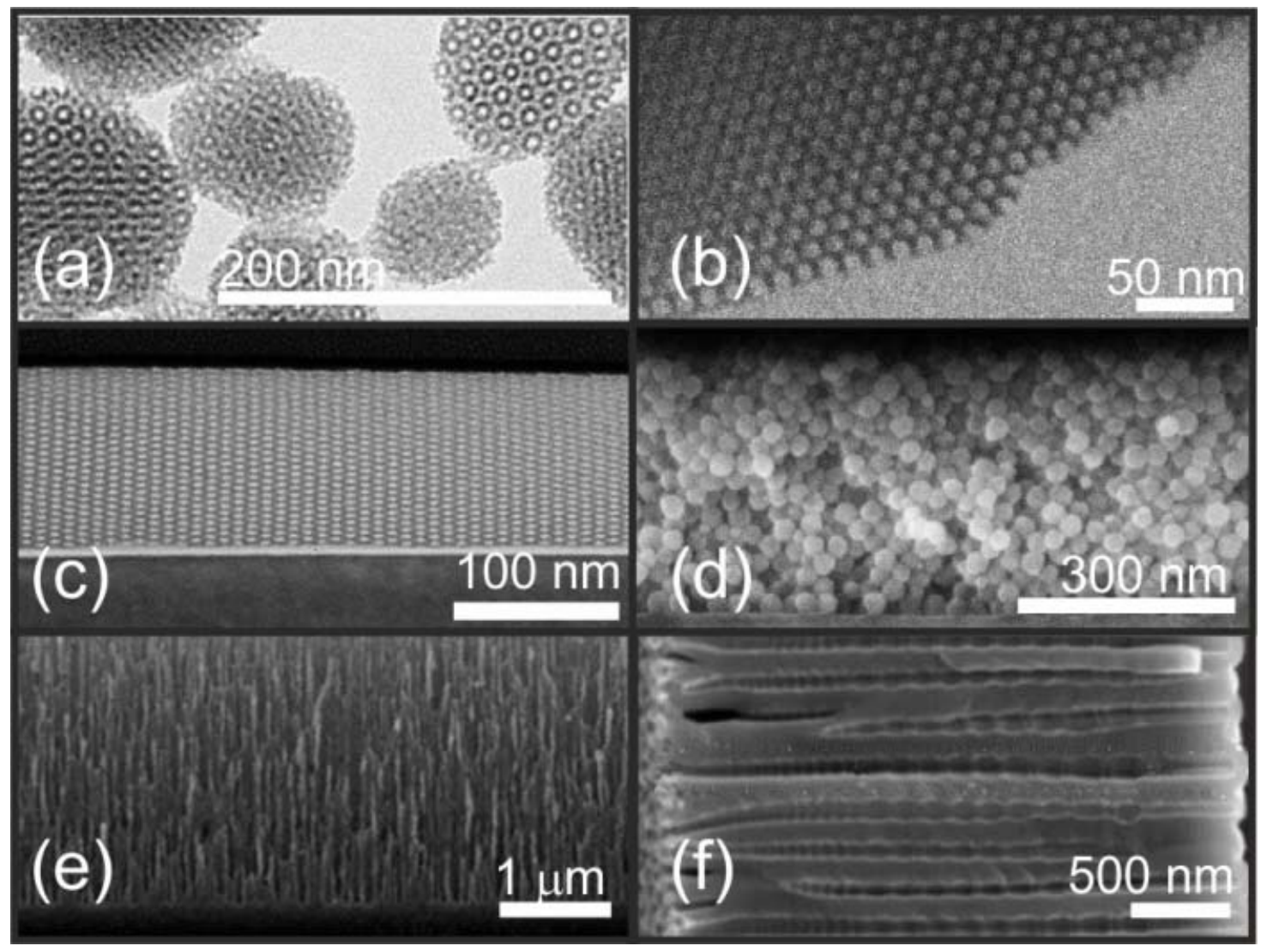

Figure 2. Examples of different porous matrices. (a) HRTEM micrograph of a mesostructured silica-templated particles (b) HRTEM image of SBA-15-template particles (rods). (c) HRTEM 
of a mesostructured film obtained from epitaxial growth of $\mathrm{SiO}_{2}$ after copolymer controlled selfassembly (d) SEM cross section of thin film based on $30 \mathrm{~nm}$ size $\mathrm{SiO}_{2}$ nanoparticle dip coating (e) SEM cross section of porous silicon prepared by electrochemical etching. (f) SEM cross section of porous $\mathrm{Al}_{2} \mathrm{O}_{3}$ structured using cyclic anodization. Reprinted with permission from (a)

${ }^{[37]}$ Copyright 2010, American Chemical Society, (b) ${ }^{[38]}$ Copyright 2016 Elsevier Ltd., (c) ${ }^{\text {[39] }}$ Copyright 2012, American Chemical Society, (d) ${ }^{[40]}$ Copyright 2012, American Chemical Society (e) ${ }^{[41]}$ Copyright 2005, American Chemical Society, (f) ${ }^{[42]}$ Copyright 2009 Wiley-VCH Verlag GmbH \& Co. KGaA, Weinheim.

nanocrystals with a narrow size distribution within the pore networks of supramolecularly templated microparticles or films, ${ }^{[15,16,18,33,43-44]}$ of packings of monodisperse nanoparticles ${ }^{[45,46]}$ or of electrochemically etched films, ${ }^{[31,47]}$ allowing a fine spectral control over the photoabsorption and photoemission resulting from quantum size confinement. Electron microscope images displayed in Figure 2 illustrate the diversity of porous materials that have been proven suitable for this purpose, which are described in more detail next. Before we continue, we would like to clarify that, in the context of this report, we use the adjective "mesostructured" to refer to a material in which there exists an array of pores ordered in a well-defined geometry, while the word "mesoporous" will be used to qualify any porous matrix regardless of the degree of ordering it may show.

\subsection{Templated metal oxide particles and films.}

Templated metal oxides constitute one of the most important families of mesostructured materials, as they display a wide range of compositions, pore sizes and network geometries. Their synthesis is versatile, with more than one established protocol and a variety of experimental conditions. The most generic synthesis involves the formation of micelles through a co-operative process in aqueous conditions. Dissolved inorganic species interact with a surfactant head-group and form a liquidcrystalline arrangement through charge matching before condensation of the inorganic phase occurs. ${ }^{[48]}$ Also, a number of other mechanisms have been proposed to describe 


\section{WILEY-VCH}

the formation of mesoporous materials in alkaline and acidic media, including nonsurfactant templates. ${ }^{[49,50]}$ This type of materials can be obtained both as particles (Fig. 1a and 1b) and films (Fig. 1c) and were the first ones used as hosts for the synthesis of perovskite nanocrystals. In 2016, the groups of Kovalenko and Yamauchi independently reported the first examples of synthesis of perovskites confined in a template made of mesostructured particles displaying well-defined pore geometries. ${ }^{[15,16]}$ In the first case, the authors used a series of commercial mesoporous $\mathrm{SiO}_{2}$ powders with cavities of different sizes and shapes (from $2.5 \mathrm{~nm}$ to $50 \mathrm{~nm}$ in diameter) which, in fact, dictate the size of the perovskite crystals grown within them. A concentrated solution of the perovskite precursors impregnates the powders and the excess is removed by filtration. Eventually, the powders were subjected to the heat treatment necessary for the evaporation of the solvent and the crystallization of the semiconductor. This method proved to be adaptable to different chemical compositions of type A cation (MA, FA, Cs) and halide anions $(\mathrm{Br}, \mathrm{I})$ and allowed authors to obtain a displacement of the emission peak up to $0.24 \mathrm{eV}$ in the green-blue region and $0.30 \mathrm{eV}$ in the red with respect to the bulk. (vide infra) In the other work, Yamauchi et al. used mesoporous $\mathrm{SiO}_{2}$ as host material, synthesized through a sacrificial organic template, to obtain $\operatorname{MAPb}\left[\mathrm{I}_{(1-\mathrm{x})} \mathrm{Br}_{\mathrm{x}}\right]_{3}$ nanocrystals in the range of $3 \mathrm{~nm}<d<7 \mathrm{~nm}$ (actually, the average pore size in the $\mathrm{SiO}_{2}$ templates). ${ }^{[16]}$ Some of these last authors also present a mesostructure in which the siliceous structure possesses a complex internal gyroidal geometry, the size of the pores being tunable between $7 \mathrm{~nm}<d<12 \mathrm{~nm}$ simply by controlling the temperature of the hydrothermal treatment. ${ }^{[43]}$ A representative electronic microscopy image of this type of system is depicted in Figure 3a.

Perovskite nanocrystals synthesized in mesostructured particles display well-defined quantum confinement effects, enhanced stability and even a lower concentration of 


\section{WILEY-VCH}

defects. However, such powders strongly diffuse light (please see inset in Figure 3a) and hence are not suitable to make optical quality (i.e., scattering free) materials, which will be required for the eventual integration in most devices such as solar cells or light emitting displays. A possible way to overcome that limitation is to impregnate porous mesostructured films with perovskite nanocrystals following any of the already mentioned methods. The first realization of $\mathrm{ABX}_{3}$ nanocrystals dispersed in an optical quality slab was achieved using a mesostructured film host made by evaporation induced self-assembly (EISA), which was infiltrated with methylammonium lead iodide $\left(\mathrm{MAPbI}_{3}\right)$ nanocrystals following a one-step synthetic method (route II). ${ }^{[18]}$ It was shown that by controlling the precursor infiltration process it is possible to prevent the formation of bulk crystals on the surface, thus yielding a film embedded exclusively with nanocrystals displaying photoabsorption and photoemission properties determined by strong exciton quantum confinement. This technique is versatile in terms of both the composition of the matrix $\left(\mathrm{TiO}_{2}\right.$ and $\left.\mathrm{SiO}_{2}\right)$ and the pore sizes available, as the use of a variety of organic structuring agents leads to final cage diameters ranging from $3 \mathrm{~nm}$ to $8 \mathrm{~nm}$. Under these conditions, the nanocrystal size distribution is mainly determined by that of the pores in the matrix, as the final dimensions of the nanoparticles are limited by that of the cage where they are synthesized and hosted. In Figure $3 b$ we show perovskite nanocrystals (dark regions) inside a network of $\mathrm{TiO}_{2}$ pores. Crystalline planes are highlighted in the image. Photoluminescence (PL) blue-shifts as large as 0.34 $\mathrm{eV}$ with respect to that of the bulk material have been observed for these confined $\mathrm{MAPbI}_{3}$ nanocrystals. The inset of Figure $3 \mathrm{~b}$ shows the red photoluminescence of an optical quality $\mathrm{TiO}_{2}$ film deposited onto a glass slide and infiltrated with $\mathrm{MAPbI}_{3}$ nanocrystals under blue excitation. The potential of this synthetic route has been recently demonstrated by Malgras et al., who have succeeded in obtaining embedded 


\section{WILEY-VCH}

$\mathrm{CsPbBr}_{3}$ perovskite nanocrystals displaying an intense blue emission from a mesostructured $\mathrm{SiO}_{2}$ film. ${ }^{[44]}$ In this example, authors propose a hierarchical de-wetting mechanism, resulting from the presence of interconnecting pore channels, to explain the appearance of a bimodal distribution of perovskite nanocrystals.

While all these approaches rely on a single step infiltration procedure, the feasibility of the two-step process (route II) to attain perovskite nanocrystals in a mesostructured matrix has also been demonstrated by Chen et al., ${ }^{[51]}$ who prepared a mixed halide perovskite such as $\mathrm{MAPbIX}_{2}$ inside a metal organic framework (MOF) film, first immersing the porous matrix in a solution of $\mathrm{PbI}_{2}$ and then dipping in a solution of MAX (methylammonium halide).

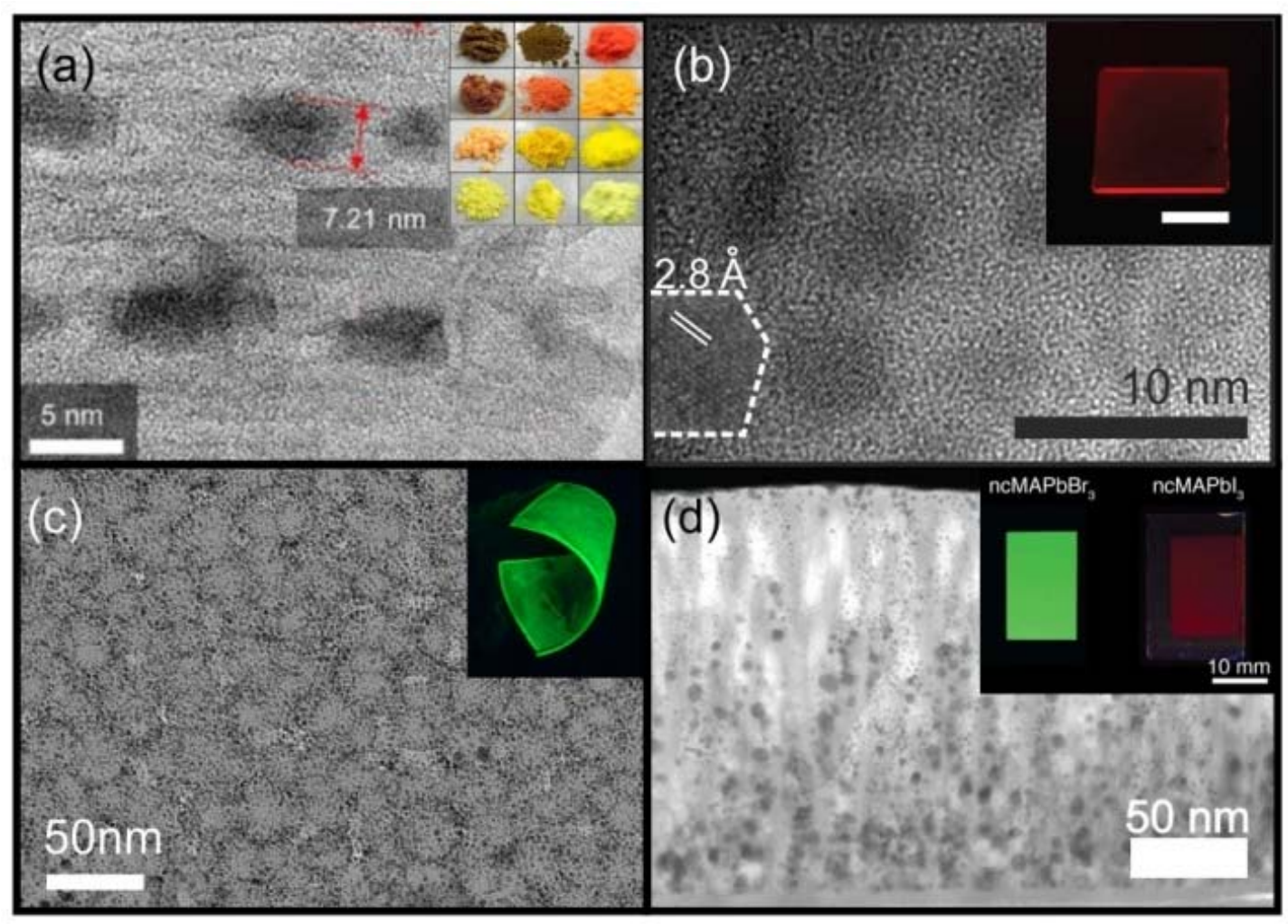

Figure 3. Electronic microscopy images of examples of porous materials containing in-situ synthesized $\mathrm{ABX}_{3}$ perovskite nanocrystals: (a) HRTEM image of porous $\mathrm{SiO}_{2}$ (pore size $7.1 \mathrm{~nm})$, dark spots are $\mathrm{MAPbBr}_{3}$ perovskite nanocrystals. Inset: photographs of $\mathrm{SiO}_{2} @$ perovskite samples with different nanocrystals size and composition. (b) HRTEM of 


\section{WILEY-VCH}

$\mathrm{TiO}_{2}$ templated mesoporous film including $\mathrm{MAPbI}_{3}$, crystallographic planes are indicated in the image. Inset: photo under UV light of the same sample. (c) STEM image of $\mathrm{SiO}_{2}$ mesoporous particulated film, dark dots belong to $\mathrm{MAPbBr}_{3}$ perovskite nanocrystals. Inset: picture of emitting flexible film made of $\mathrm{SiO}_{2}$ nanoparticles, $\mathrm{MAPbBr}_{3}$ nanocrystals and PDMS. (d) Bright Field-STEM image of nanoporous $\mathrm{Al}_{2} \mathrm{O}_{3}$ (pore size $\cong 7 \mathrm{~nm}$ ) including $\mathrm{CsPbX}_{3}$ nanocrystals. Inset: photographs of AAO thin films including perovskite nanocrystals exposed to ultraviolet illumination. Reproduced with permission. (a) ${ }^{[16]}$ Copyright 2016, American Chemical Society (b) ${ }^{[18]}$ Copyright 2017, Wiley-VCH. (c) ${ }^{[45]}$ Copyright 2018, American Chemical Society (d) ${ }^{[31]}$ Copyright 2017, AAAS

\subsection{Nanoparticle packings}

Films made by packing nanoparticles show a network of accessible interconnected pores that can also be used to infiltrate perovskite nanocrystals. These matrices can be obtained by means of different deposition techniques, such as dip-coating, spin-coating, doctor blade, Langmuir-Blodgett, or spray-pyrolysis with thicknesses comprised between the tens to hundreds of nanometers. ${ }^{52-56}$ Nanoparticle packings, in spite of being disordered, show an interstitial volume that possesses several features in common with that of ordered mesostructures, such as a well-defined pore size distribution. ${ }^{[40]}$ However, in this case, pores cannot be considered as well-defined cages, as opposed to the case of mesostructured materials, as they present a much higher and more irregular interconnection. Figure 3(c) shows a characteristic cross-section of this kind of mesoporous film in which perovskites nanocrystals are synthesized using a one-step method.

By following any of the routes described in Figure 1, it is possible to obtain perovskite nanocrystals deposited onto the nanoparticle walls and homogeneously dispersed throughout the matrices. Interestingly, it is still possible to finely control the size of nanocrystals synthesized within these matrices even when they are typically much smaller than the average interstitial size. This can be explained on the basis of the 


\section{WILEY-VCH}

hypothesis that pores act as nanoreactors in which the diffusion of reactants is limited by the tortuosity of the network. Under these conditions, a clear correlation is found between the concentration of reactants and the nanocrystal size attained for a matrix of fixed interstitial network. The intricate porosity of the lattice hinders diffusion and thus indirectly limits crystal growth, although not through its geometry as in the case of the mesostructured films discussed in the previous section. The fact that the pore volume is much larger than the nanocrystals formed within open the door to further processing of these films, which has allowed the preparation of flexible films displaying enhanced and stable photoluminescence from embedded perovskite quantum dots (see inset in Figure 3c). ${ }^{[45]}$ Similar infiltration procedures (route I) have been followed to prepare $\mathrm{ABX}_{3}$ nanocrystals within porous films of F-doped $\mathrm{TiO}_{2}$ particles ${ }^{[57]}$ and polydimethylsiloxane (PDMS) ${ }^{[33]}$ While the former relies on the acid etching of a film to obtain porosity, the latter makes use of the embedding and subsequent elimination with aqua regia of gold nanoparticles in the polymeric matrix. In these two approaches optical quality (transparency) of the host films is not granted.

\subsection{Electrochemically etched porous matrices}

Porous silicon ( $\mathrm{Si}$ ) and aluminum oxide $\left(\mathrm{Al}_{2} \mathrm{O}_{3}\right)$ films can be obtained by electrochemical etching of wafers or aluminum foils using well established methods that allow a fine control over their internal pore structure. One of the most relevant characteristic of these materials is that they present open tubular pores of large aspect ratio that run along the whole thickness of the film, although other pore geometries can be prepared by tuning the electrochemical parameters. Demchyshyn et al. have employed these type of matrices to synthesize perovskites within. ${ }^{[31]}$ Typical pore sizes are in the range of the few tens of nanometers and can be controlled by tuning the electrochemical parameters. The versatility of this approach has been demonstrated by 


\section{WILEY-VCH}

infiltrating perovskite nanocrystals of different compositions ( $\mathrm{MAPbI}_{3}, \mathrm{MAPbBr}_{3}$, $\mathrm{CsPBr}_{3}$ and $\mathrm{MAPbCl}_{3}$ ). A typical image of a cross-section of a porous anodized alumina structure impregnated with perovskite crystallites is shown in Figure 3d. More recently, Ghosh et al. succeeded in confining $\mathrm{MAPbI}_{3}$ crystallites following synthetic route I and employing as a host an array of nanotubes prepared by silver-assisted chemical etching of a silicon wafer. ${ }^{[47]}$ Nanocrystals are deposited onto the silicon walls and their size is controlled with the degree of dilution of the precursor solution. Jana et al. used a twostep method to infiltrate the wormhole mesoporous film of $\mathrm{Al}_{2} \mathrm{O}_{3}$ to obtain perovskite nanocrystals of $3.6 \mathrm{~nm}$ (average) with tunable emission. ${ }^{[58]}$

\section{Optoelectronic properties}

\subsection{Quantum confinement effects and stability.}

One of the most characteristic features of nanocrystalline semiconductors is the gradual increase of the electronic bandgap with decreasing particle size resulting from the quantum confinement of the exciton, ${ }^{[59]}$ which consequently gives rise to a spectral blue-shift of photoabsorption and photoemission. In Figures $4 \mathbf{a}$ and $4 \mathrm{~b}$, we show different examples of this behavior for different perovskite nanocrystals synthesized within porous matrices. ${ }^{[16,18,47,60]}$ Just like it has been done for other semiconductors, the dependence of the bandgap of perovskites synthesized within porous matrices with size have been described using Brus equation:[61]

$$
E_{g, n c} \approx E_{g, b u l k}+\frac{h^{2}}{8 \mu R^{2}}-\frac{1.786 e^{2}}{4 \pi \varepsilon_{0} \varepsilon_{r} R}
$$

which, based on a hydrogenic model, describes the interaction between electron-hole pairs strongly confined in spherical semiconductor nanocrystals. ${ }^{[62]}$ In equation (1), $E_{g, n c}$ is the exciton lowest excited energy state within the nanocrystal, $E_{g}$, bulk is the bandgap 


\section{WILEY-VCH}

of the bulk perovskite, $R$ is the radius of the nanoparticle, $h$ is Planck's constant, $\mu$ is the exciton reduced mass, $e$ is the electron charge, and $\varepsilon_{0}$ and $\varepsilon_{\mathrm{r}}$ are the dielectric constants of vacuum and the perovskite, respectively. The second term on the right side of the equation corresponds to the first energy level of a quasiparticle of reduced mass $\mu$ confined in a spherical well of radius $R$ limited by a wall of infinite potential. The third term is the Coulomb energy, which results from the electrostatic interaction between electron and hole within the nanocrystal. The use of Brus formula implies a series of approximations that may not be strictly valid for smallest crystal sizes.

A summary of reported values of $E_{\mathrm{g}, \mathrm{nc}} v s$. $R$, extracted from six different publications, is plotted in Figure 4c, along with the behavior predicted by Brus formula for both $\mathrm{MAPbI}_{3}$ and $\mathrm{MAPbBr}_{3}$ (red solid and green dotted lines respectively). ${ }^{[15,16,18,43-45]}$ It should be remarked that the approaches based on the synthesis of nanocrystals within porous matrices typically lead to $\mathrm{ABX}_{3}$ nanocrystals with sizes smaller than the reported exciton Bohr radius, ${ }^{[63]}$ those being $\mathrm{R}_{\mathrm{B}, \mathrm{MAPbI} 3}, \approx 3 \mathrm{~nm}, \mathrm{R}_{\mathrm{B}, \mathrm{MAPbBr} 3,} \approx 2 \mathrm{~nm} .{ }^{[64]}$ and $\mathrm{R}_{\mathrm{B}, \mathrm{CsPbBr} 3}, \approx 3.5 \mathrm{~nm},{ }^{[65]}$ and hence strong quantum confinement effects are observed in most cases. Consequently, some of the largest luminescence spectral shifts have been reported for perovskite nanocrystals synthesized within porous matrices. ${ }^{[15,16,18,44]}$ Also, analysis of the full width at half maximum of the emission peaks reveals that the inhomogeneous broadening is similar to that observed in colloidal nanocrystals, which implies that the distribution of sizes is equally precisely controlled. (for instance, compare values in ref. 15)

Enhanced stability is an unexpected beneficial side effect of confinement. Spatially restrained perovskite nanocrystals do not show strong impairment when exposed to ambient conditions as it happens for non-encapsulated $\mathrm{ABX}_{3}$ homogeneous film. This is exemplified in Figure 4d, where the time evolution of the PL intensities measured from 


\section{WILEY-VCH}

a bulk film made of $\mathrm{CsPbBr}_{3}$ and from meso- $\mathrm{SiO}_{2}$ particles embedded nanocrystals of similar composition are compared. The matrix walls and the tortuosity of the pore network may hinder the diffusion of degrading gases (principally $\mathrm{O}_{2}{ }^{[66,67]}$ and $\left.\mathrm{H}_{2} \mathrm{O}^{[68,69]}\right)$. In the case described by Wang et al., for example, ${ }^{[17]}$ a silicon-based matrix was used in which ex-situ pre-formed $\mathrm{CsPbBr}_{3}$ nanocrystals were infiltrated to increase stability and to limit their anionic segregation. These integrated systems have shown an improvement in stability against temperature and light. After $96 \mathrm{~h}$ under $\lambda=405 \mathrm{~nm}$ laser illumination, the perovskites maintained $80 \%$ of the emission intensity, unlike the nonintegrated crystals, which lost $60 \%$. Likewise, integrating these systems into polymeric matrices can increase the resistance to the environment ${ }^{[34,35,45,70]}$ preserving the optical properties of the nanocrystals. This sheltering effect occurs for all nanocrystals regardless the method (routes I, II or III) used to embed them in the matrix. It has also been shown that confined nanocrystals also present excellent photoluminescence performance under 96h UV light exposure. ${ }^{[17]}$ 

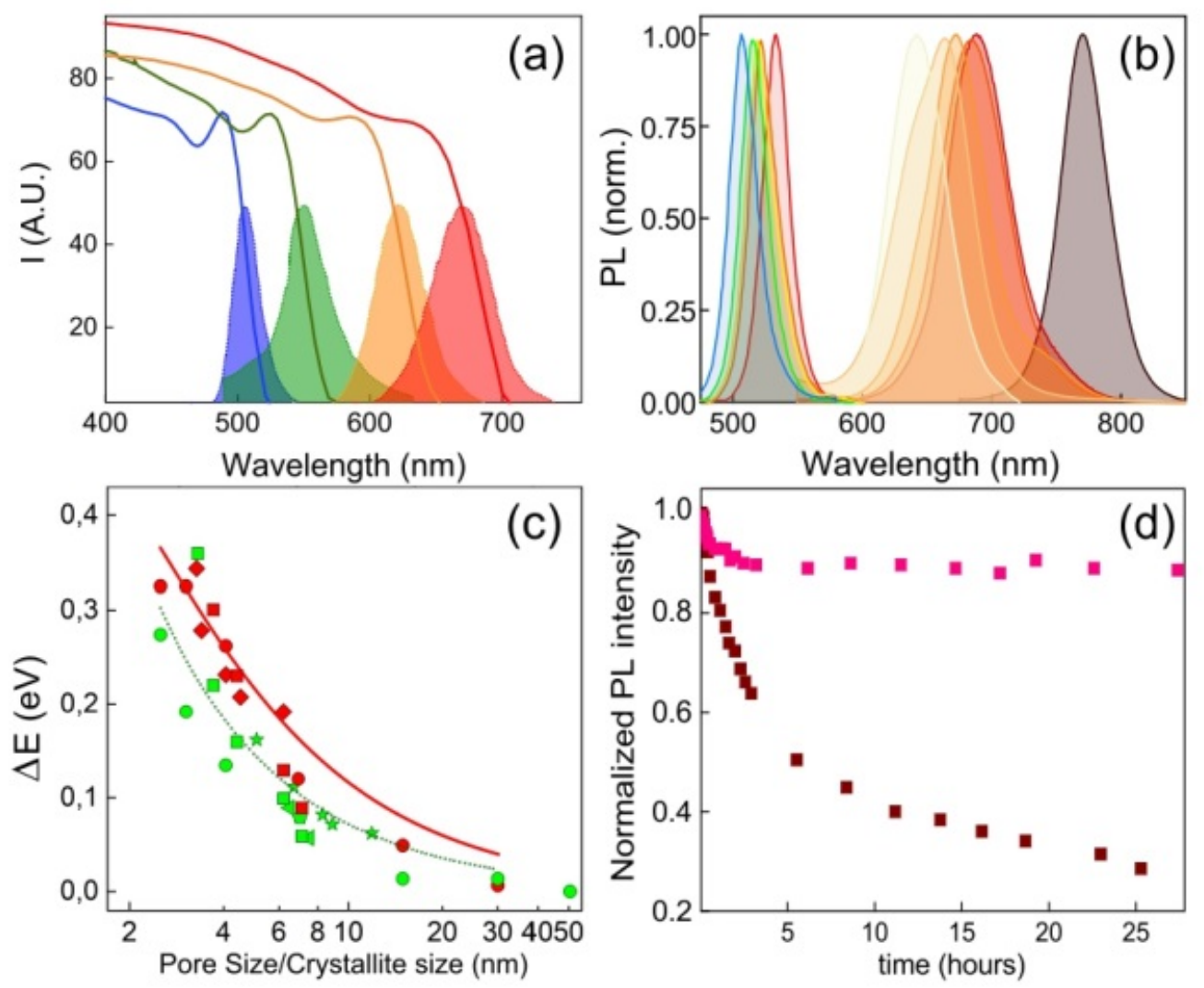

Figure 4. (a) Absorption and emission spectra of nc-MAPb $\left[\mathrm{I}_{(1-x)} \mathrm{Br}_{\mathrm{x}}\right]_{3}$ in $\mathrm{SiO}_{2}$ particles with $\mathrm{x}=0.0,0.3,0.6$ and 1.0 (red, orange, green, blue, respectively), data extracted from reference [16]. (b) Emission spectra $(480 \mathrm{~nm}<\lambda<550 \mathrm{~nm})$ of $\mathrm{nc}-\mathrm{MAPbBr}$, synthesized within a film of packed $\mathrm{SiO}_{2}$ particles with sizes (in $\mathrm{nm}$ ) of 11.9 (red line), 8.9 (orange line), 8.3 (yellow line), 6.7 (green line), 5.1 (blue line); emission spectra of nc-MAPbI $3(600 \mathrm{~nm}<\lambda<820 \mathrm{~nm}$ ) synthesized within mesostructured $\mathrm{TiO}_{2}$ films with sizes of (in nm): 6.2 (dark red line), 4.5 (red line), 4.1 (dark orange line), 3.4 (orange line) and 3.3 (light grey line), data extracted from reference [16] and [18]. The emission spectrum of a bulk film is also plotted for comparison (brown line, grey shaded). (c) Band gap shift, $\Delta E_{g}=E_{g, n c}-E_{g, b u l k}$, estimated from the position of the photoemission spectra maxima, as a function of the experimentally determined crystallite size. Data adapted from [15] (green and red circles), [43] (green stars), [16] (green and red square), [18] (red diamond), [44] (green pentagon), [45] (green triangles). Solid lines are the expected trends after Brus equation for both $\mathrm{MAPbBr}_{3}$ and $\mathrm{MAPbI}_{3}$ perovskite nanocrystals (green and red lines, respectively). (d) Evolution of the normalized photoluminescence during 24 hours of illumination of a bulk film (brown squares) and encapsulated nanocrystals (pink squares). Data adapted from ref. [31].

\subsection{Decay dynamics and photoluminescence quantum yield.}

In general, excited states of semiconductor nanocrystals present a shorter lifetime than the bulk version of the same material. This applies also to lead halide perovskites embedded in porous matrices. However, in this latter case, the energy of the electronic 


\section{WILEY-VCH}

bands of the host strongly affects recombination, as electrons and holes could be transferred to the matrix, providing non-radiative decay paths. In Figure 5a, we represent the log-log decay curves measured for $\mathrm{MAPbI}_{3}$ nanocrystals of the same size (4.5 nm) embedded in $\mathrm{SiO}_{2}$ and $\mathrm{TiO}_{2}$. Results for a bulk film are also shown for the sake of comparison. Lifetimes of nanocrystals confined in a matrix based on $\mathrm{TiO}_{2}$ and $\mathrm{SiO}_{2}$ templated with the same pore size may present decay rates two orders of magnitude larger than that of a bulk film. The differences in lifetime between both collections of nanocrystals can be explained in terms of the possibility to inject charge carriers to the conduction (valence) band of the metal oxide matrix. In the case of $\mathrm{TiO}_{2}$ matrix, $\mathrm{ABX}_{3}$ nanocrystals are able to inject electrons into the conduction band, as schematized in Figure $5 \mathrm{~b}$ (left), while for the case of the $\mathrm{SiO}_{2}$-based host this nonradiative decay path is not available, Figure $5 \mathrm{~b}$ (right). Please notice that nanocrystals deposited onto the inner walls of a porous matrix by means of synthetic routes I or II present no steric bulky organic ligands. On the other hand, functionalization of the pore walls is an alternative means to explore the potential of the interaction of the nanocrystals with different chemical species that can act as surface trap passivating agents, a possibility that has not been explored so far. The effect is particularly evident in cases where polymer matrices are used, in which the lifetimes are comparable with those of the bulk material. ${ }^{[70]}$ In this regard, further research should be carried out on the effect of residual solvent molecules normally used in the synthesis of perovskites, which could remain adsorbed or somehow coordinated to the pore walls and therefore act as a coordination site. ${ }^{[27]}$ In Figure 5c we compile the lifetimes that have been reported for nanocrystalline systems synthesized within porous matrices. Interestingly, the lifetime vs. size curve peaks for a crystal size of around $7 \mathrm{~nm}$. This sort of "magic size" behavior has been reported for other semiconductor nanocrystals. ${ }^{[71]}$ A complete understanding of 
this feature will require a deeper analysis of the evolution with crystal size of the density of deep and shallow traps as well as of their relative energy with respect to the bottom and top of valence and conduction band, respectively.
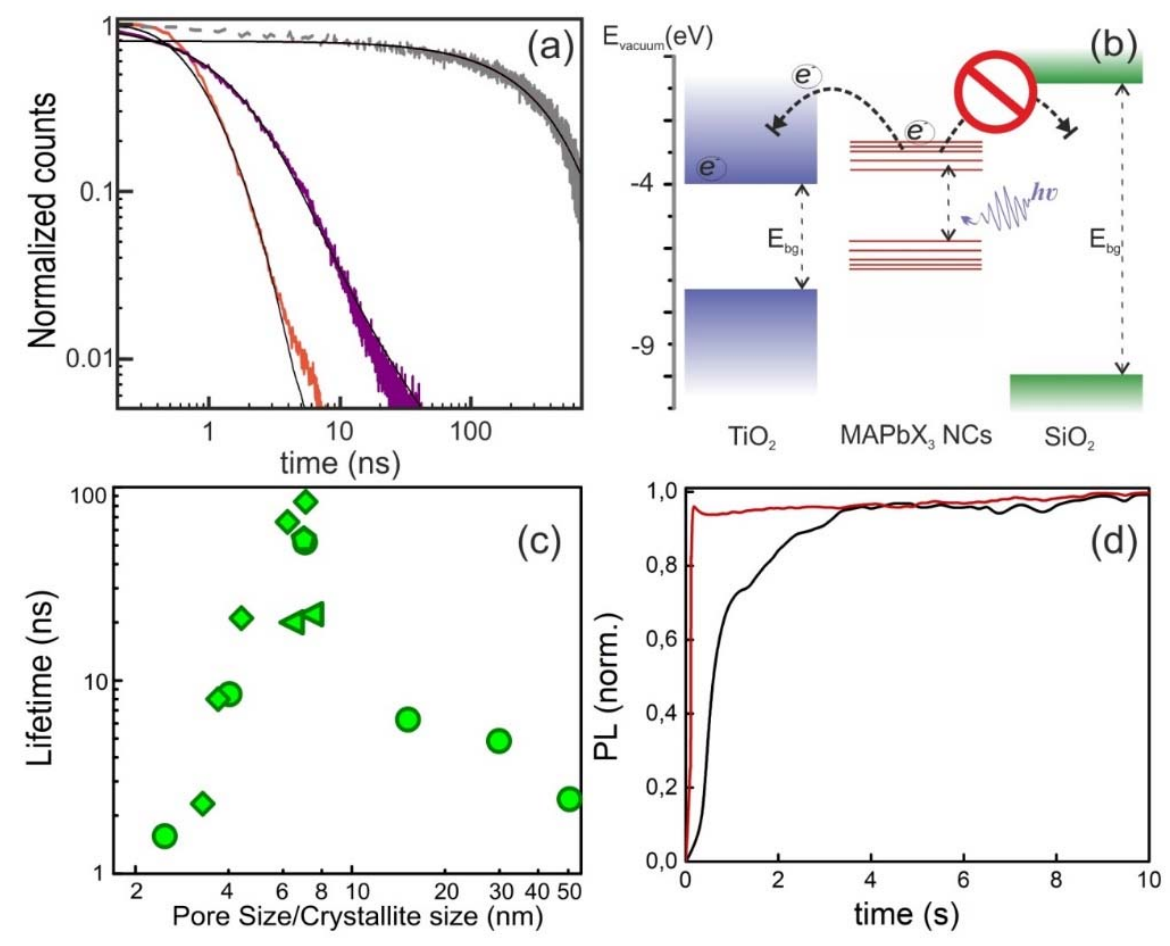

Figure 5. (a) Normalized photoluminescence decay obtained from perovskite nanocrystals templated in $\mathrm{SiO}_{2}$ (violet line) and $\mathrm{TiO}_{2}$ (orange line) matrices with the same pore size distribution and containing $\mathrm{MAPbI}_{3}$ perovskite nanocrystals of the same size. Black line represents the data obtained for a bulk film. (b) Electrons injection scheme of a $\mathrm{TiO}_{2}$ or $\mathrm{SiO}_{2} @$ perovskite $\left(\mathrm{MAPbX}_{3}\right)$ structure (c) Lifetime measured for different perovskite nanocrystals templated in porous systems confined perovskite as function of pore size of the host structure or the crystallite size. Data adapted from [15] (green circles), [16] (green diamond), [44] (green pentagon),[45] (green triangles). (d) Evolution of the normalized photoluminescence against time for nanocrystal templated system (red line) and bulk system (black line) at initial illumination Reproduced with permission from (a) and (d) ${ }^{[18]}$ Copyright 2017, Wiley-VCH

Apart from the enhanced environmental stability mentioned in the previous section, perovskite nanocrystals confined in porous matrices present some clear advantages with respect to bulk films as far as optical performance is concerned. One particularly interesting feature of porous hosted perovskite nanocrystals is that they present a much 


\section{WILEY-VCH}

faster activation of the photoluminescence than that of their bulk counterparts as we show in Figure 5d. Although It is well known that the PL of organic lead halides is activated when they are photo-excited in the presence of molecular oxygen ${ }^{72}$ and the mechanism responsible for this activation has been thoroughly studied and largely debated, ${ }^{[73]}$ the typical time scale involved in this activation for bulk organic lead halides is orders of magnitude longer than that observed for nanocrystals pumped using similar excitation intensities. This observation suggests that the large surface-to-volume ration characteristic of nanocrystals favour a much faster defect passivation/annihilation than in their bulk counterparts. This is in good agreement with the hypotheses that assume that modifications occurring at the surface trigger defect annihilation in the bulk of the material. ${ }^{[67,74]}$

A possible explanation of this effect can be related to the small size of the confined perovskite crystals. As lattice defects (surface, interstitial, vacancies) normally constitute recombination channels that quench the PL, the photo-induced ion migration that occurs in these perovskite compounds can promote the deactivation/passivation of these non-radiative paths. In consequence, this annihilation effect is much faster leading, consequently, to a quick stabilization of the PL. It is important to mention that this proposed mechanism is different from what is postulated in colloidal perovskites where ligands act as a brake on the photoinduced ion migration. ${ }^{[75]}$

Another remarkable property of confinement is that it allows attaining films in which the high PLQY expected for semiconductor quantum dots is fully preserved. Bear in mind that the very significant drop of PLQY of colloidal perovskite nanocrystals when they are assembled in a film is one of the main obstacles towards the development of optoelectronics based on them. ${ }^{[76,77]}$ This will also strongly depend on how feasible the injection and extraction of charges from the ensemble is, a subject that is discussed in section 4.4. In fact, it should be noted that most of the nanocrystalline systems described 


\section{WILEY-VCH}

in this Progress Report present PLQY values higher than their bulk counterparts. Values of PLQY above $50 \%$ can be attained for a wide range of sizes and using different porous film matrices. This opens up optimistic perspectives for the further development and implementation of the approach herein described in actual devices. An analysis of the PLQY values in combination with the dynamics of the PL decay can provide some insight about the radiative and non-radiative recombination and hence about the trap states or defects present in nanocrystals.

With respect to the effect of traps, theoretical calculations envisage that the most energetically favorable defects in the bulk form of this type of compounds are vacancies of $\mathrm{A}$ and $\mathrm{X}$ sites. ${ }^{[78]}$ These sites are normally considered as shallow traps and do not affect the electron transport or the radiative recombination. However, the predictions for nanocrystals are rather different. For instance, interstitial defects at the surface are the most stable ones when $\mathrm{CsPbBr}_{3}$ nanocrystals are considered, ${ }^{[79]}$ hence pointing as passivation as a way to minimize non-radiative channels. While for perovskite nanocrystals synthesized in solution the role of ligands is both passivating defects and stabilizing the colloid, for nanostructured perovskites prepared in porous matrices the casuistic is more complex. To start, part of their surface area is in contact with the host walls and the rest with the ambient of the pore cavity. Also, remnant solvent molecules on the semiconductor surface may play a non-predesigned passivating role, and charge carriers may be transferred to the matrix depending on the relative position of the electronic bandgap of the host and that of the perovskite. In spite of this complexity, observations suggest that the role of the host matrix might be beneficial: different authors have reported that nanocrystals synthesized within mesoporous matrices may present either a lower density or a better harnessing of defects. ${ }^{[15,44]}$ In this regard, Prieto et al. have shed some light on this matter suggesting functional groups on the host walls 


\section{WILEY-VCH}

can passivate surface defects in perovskite nanocrystals depending on their acidity. Thus, aluminate groups present in mesoporous alumina will harness exposed $\mathrm{Pb}$ ion defects in MAPbBr3 nanocrystals synthesized within the pores, while this effect may not occur when silanol groups are present, as it is the case on mesoporous $\mathrm{SiO}_{2} .^{[80]}$

\subsection{Reported data compendium and summary.}

We provide a summary of the main structural and optical features of the different matrices in Tables 1 and 2 (powders: Table 1 and films: Table 2). In both tables, we compiled the different approaches employed to attain $\mathrm{ABX}_{3}$ nanocrystals within their porous networks, along with the characteristic photoemission peak attained in this case and the reported PLQY values. 
WILEY-VCH

\begin{tabular}{|c|c|c|c|c|c|c|}
\hline Host & guest & pore size $(\mathrm{nm})$ & $\begin{array}{l}\lambda_{\max } \\
(\mathrm{nm}) \\
\end{array}$ & $\begin{array}{l}\lambda_{\text {bulk }} \\
(\mathrm{nm})\end{array}$ & QY\% & ref. \\
\hline MSU-H & 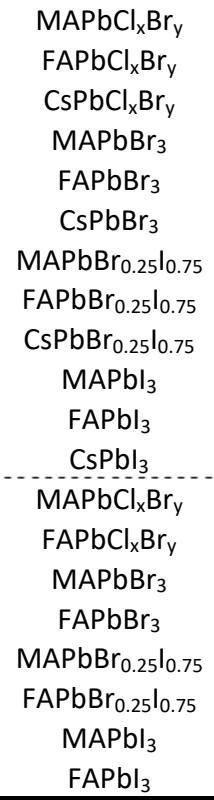 & 3 & $\begin{array}{l}510 \\
495 \\
/ \\
540 \\
520 \\
520 \\
660 \\
640 \\
630 \\
700 \\
705 \\
665 \\
-475 \\
475 \\
470 \\
490 \\
600 \\
600 \\
625 \\
630\end{array}$ & $\begin{array}{l}1 \\
1 \\
1 \\
1 \\
1 \\
1 \\
1 \\
1 \\
1 \\
1 \\
1 \\
1 \\
1 \\
1 \\
1 \\
1 \\
1 \\
1 \\
1 \\
1\end{array}$ & \begin{tabular}{c}
20 \\
15 \\
$/$ \\
22 \\
36 \\
48 \\
2 \\
8 \\
15 \\
8 \\
15 \\
4 \\
\hdashline 25 \\
10 \\
8 \\
16 \\
10 \\
8 \\
2 \\
5
\end{tabular} & 15 \\
\hline $\begin{array}{l}\text { meso-SiO } \\
\text { (MCM-41) }\end{array}$ & $\mathrm{MAPbBr}_{2} \mathrm{I}_{1}$ & $\begin{array}{l}7.1 \\
6.2 \\
4.4 \\
3.7 \\
3.3 \\
-7.1 \\
6.2 \\
4.4 \\
3.7 \\
3.3 \\
-7.1 \\
6.2 \\
4.4 \\
3.7 \\
3.3 \\
-7.1 \\
6.2 \\
4.4 \\
3.7 \\
3.3\end{array}$ & $\begin{array}{l}525 \\
517 \\
504 \\
492 \\
466 \\
-571 \\
569 \\
551 \\
523 \\
510 \\
-642 \\
636 \\
620 \\
590 \\
559 \\
721 \\
705 \\
667 \\
642 \\
585\end{array}$ & 729 & $\begin{array}{l}2.9 \\
3.7 \\
5.5 \\
2.2 \\
1.2 \\
1 \\
1 \\
1 \\
1 \\
1 \\
1 \\
1 \\
1 \\
1 \\
1 \\
1 \\
1 \\
1 \\
1 \\
1\end{array}$ & 16 \\
\hline KIT-6 & $\mathrm{MAPbBr}_{3}$ & $\begin{array}{c}11.9 \\
8.9 \\
8.3 \\
6.7 \\
5.1 \\
11.9 \\
8.9 \\
8.3 \\
6.7 \\
5.1\end{array}$ & $\begin{array}{l}544 \\
541 \\
539 \\
532 \\
521 \\
7765 \\
752 \\
747 \\
734 \\
708\end{array}$ & 559 & $\begin{array}{l}1 \\
1 \\
1 \\
1 \\
1 \\
1 \\
1 \\
1 \\
1 \\
1\end{array}$ & 43 \\
\hline SBA 15 & $\mathrm{CsPBr}_{3}$ & 1 & 507 & 525 & 4.1 & 44 \\
\hline
\end{tabular}

Table 1. Powders: type of host (matrix) guest ( $\mathrm{ABX}_{3}$ perovskite), pore size, spectral position of the maximum emission, spectral position of bulk, and photoluminescence quantum yield for powders fabrication. 


\section{WILEY-VCH}

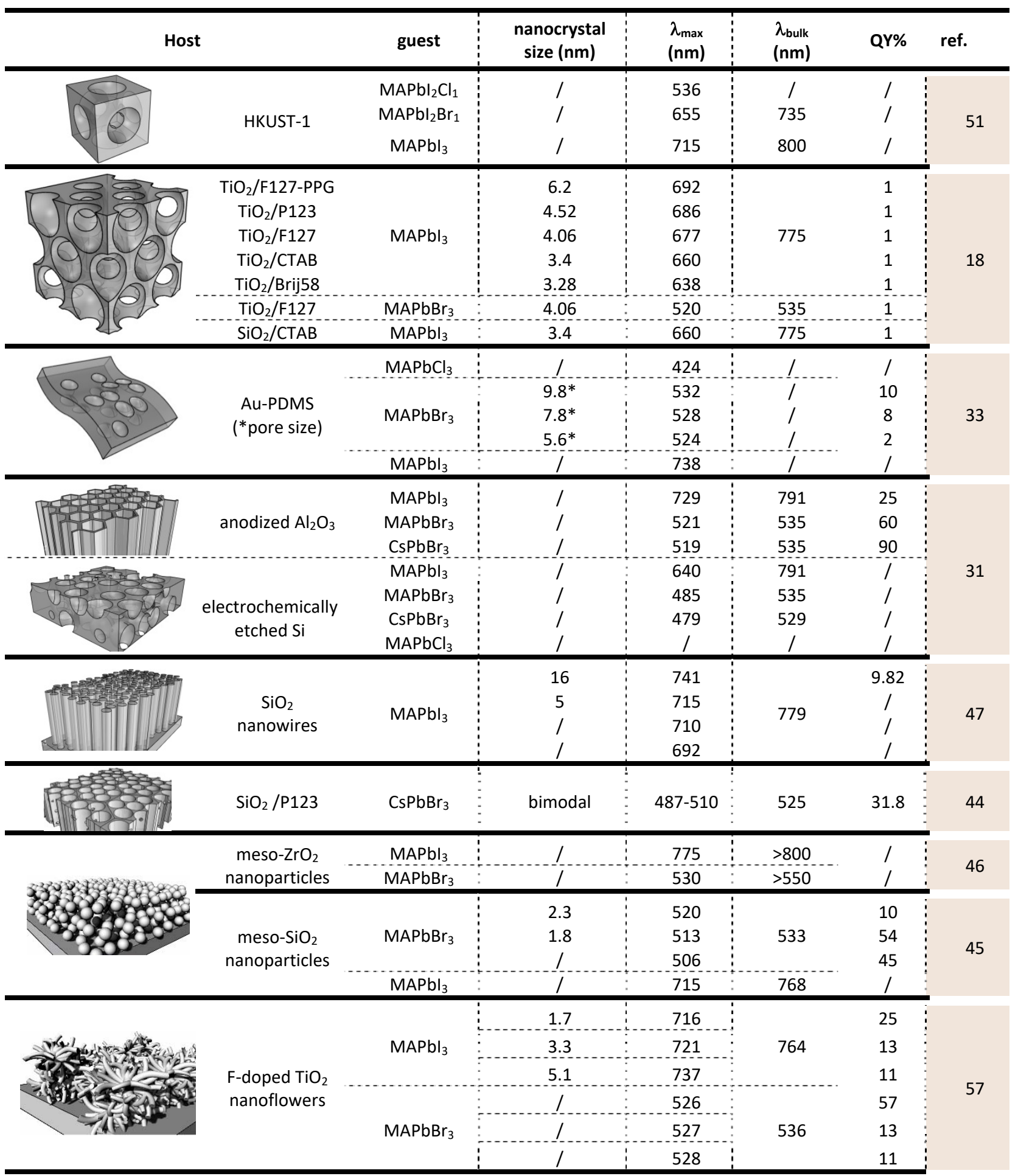

Table 2. Films: type of host (matrix) guest ( $\mathrm{ABX}_{3}$ perovskite), perovskite nanocrystals size, spectral position of the maximum emission, spectral position of bulk, and photoluminescence quantum yield for thin films fabrication. 


\section{WILEY-VCH}

\subsection{Integration in devices.}

There are several configurations in which the host-guest materials herein described could be integrated into photovoltaic or light emitting devices. Charge transport through the ensemble will be determined by the conducting or insulating nature of the matrix, as well as by the efficiency of charge transfer between the host and the nanocrystals. In fact, if the density of nanocrystals on the pore walls is large enough, charge percolation could also eventually give rise to conduction through the perovskite, providing an alternative means for injection and extraction. ${ }^{[81]}$ As mentioned before, for the case of $\mathrm{TiO}_{2}$ matrices $\mathrm{ABX}_{3}$ nanocrystals are able to inject photoelectrons to the conduction band of the metal oxide. This mechanism allowed Lee et al. to develop the first example of a sensitized solar cell using $\mathrm{MAPbI}_{3}$ nanocrystals, which were synthesized directly into a mesoporous $\mathrm{TiO}_{2}$ thin film. ${ }^{[46]}$ This solar cell, whose structure is depicted in Figure 6a, displayed an efficiency of $6.23 \%$ similar to identical thickness sensitized with an organic dye, which encourages further research. Even at this stage, this type of nanostructured perovskite solar cell architecture can find applications in semitransparent or colored devices. ${ }^{[5]}$ Regarding lighting applications, it was demonstrated that $\mathrm{MAPbI}_{3}$ and $\mathrm{CsPbBr}_{3}$ synthesized within porous columnar $\mathrm{Al}_{2} \mathrm{O}_{3}$ results in electrically addressable nanocrystals that hence form the active layer of a supramolecular diode (LED) with the architecture shown in Figure $6 b^{[31]}$ The low efficiency of these devices compared with the high PLQY values of the nanocrystals indicates that there is still a long way to improve the matrix- $\mathrm{ABX}_{3}$ nanocrystal band junction engineering. An alternative proposed in the work of Vassiliakopoulu et al. ${ }^{[82]}$ is a sort of bandgap engineering where they use 2D perovskites infiltrated into the porous system together with nanocrystals to favor the transfer of energy from the higher bandgap materials towards the latter. From a different perspective, perovskite nanocrystals could also be 


\section{WILEY-VCH}

used as color converting coatings by adapting them to the shell of a bulb, as it has been recently shown. ${ }^{[4]}$ In this case, the control of the size of the perovskite nanocrystals inside the pore network of the nanoparticles matrix leaves enough room for the infiltration of a polymer. Under the adequate conditions, this process leads to flexible and adaptable films in which the high PLQY of the particles is preserved. This application is illustrated in Figure 6c, where a GaN LED is cover with one of this flexible polymer-based film to reproduce the spectra shown in the inset. ${ }^{[45]}$
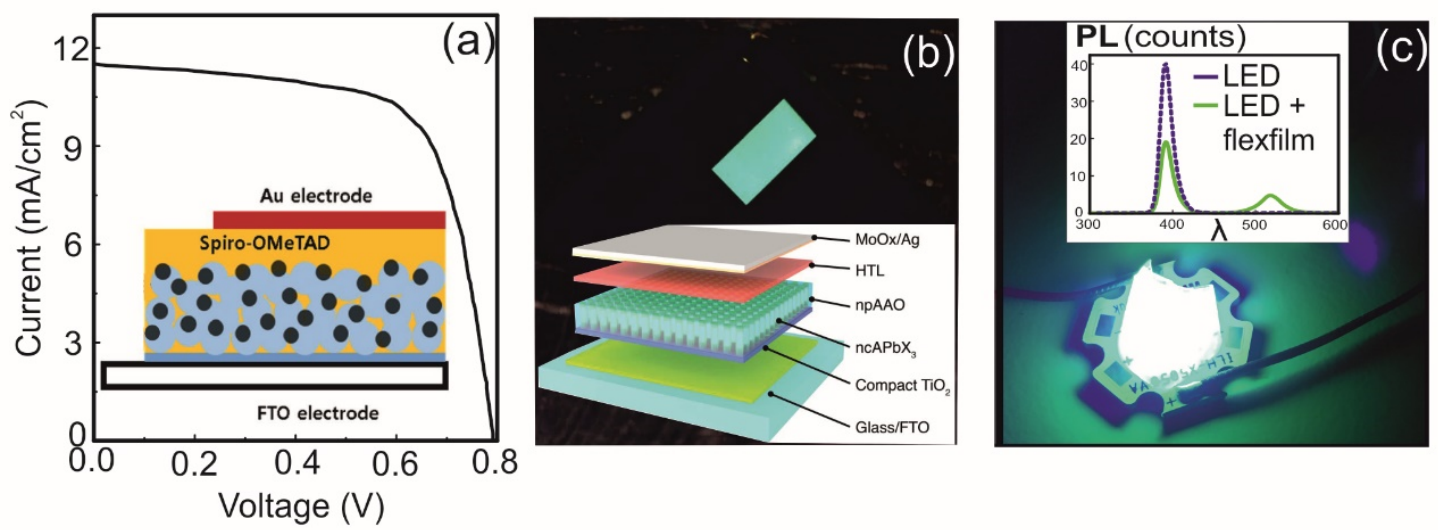

Figure 6. (a) $\mathrm{J}-\mathrm{V}$ curve from the $\mathrm{DSSC}$ based on $\mathrm{MAPbI}_{3}$ nanocrystals embedded in a mesoporous $\mathrm{TiO}_{2}$ layer. (Inset: architecture of the DSSC). (b) Image obtained from an operating $\mathrm{CsPBr}_{3}$ LED based on nanocrystals embedded in $\mathrm{Al}_{2} \mathrm{O}_{3}$ porous system. (Inset: architecture of the LED). (c) Image obtained from a $\operatorname{GaN} \operatorname{LED}\left(\lambda_{\mathrm{em}}=405 \mathrm{~nm}\right)$ covered with a flexible film containing $\mathrm{MAPbBr}_{3}$ nanocrystals embedded in a nanoparticulated $\mathrm{SiO}_{2}$ matrix with $\mathrm{PDMS}$. Inset: PL emission before and after the color conversion. Reproduced with permission (a) ${ }^{[46]}$ Copyright 2018, Wiley-VCH, (b) ${ }^{[31]}$ Copyright 2017, AAAS, (c) ${ }^{[45]}$ Copyright 2018, American Chemical Society

\section{Outlook}

In this report, we have reviewed the recent progress in the development and applications of organic/inorganic lead halide perovskites embedded within matrices in which there exists a strict control over their porous structure. The different methods that have been followed to integrate perovskite nanocrystals of controlled size in the 


\section{WILEY-VCH}

interstices of a material, and how the specific characteristics of these voids affect the outcome of the process, have been reviewed and classified in three different routes. The different examples provided show the great potential of this approach to improve several key aspects of nanostructured perovskites and the devices based on them, such as environmental stability, performance, activation time, versatility and adaptability. Although the results so far obtained constitute more a proof of concept than a definitive evidence of the viability of this approach, they are promising enough to encourage further research on this topic. Leaving aside the sheltering effect the matrices have and the ease of processing they offer, probably the most relevant feature of the approach herein discussed is the possibility of attaining films containing a nanocrystalline version of the semiconductor preserving high absorption and emission efficiency. It can be foreseen that the optimization of charge injection in these systems will be a relevant subject of research in this field in the near future. From a different perspective, the inner walls of porous networks can act as substrates for anchoring different types of organic functionalities that can help to further stabilize and improve the light harvesting and emission properties of the perovskite nanocrystal guests, as well as to controllably modify them by careful design of the interplay between perovskite nanocrystals and the functional groups. The wide variety of phenomena that can arise from this interplay should also trigger investigations along this line.

Most of the cases examined so far concern porous silica-based structures which, due to the distinctive bending quality of the $\mathrm{O}-\mathrm{Si}-\mathrm{O}$ bond, allowed a preponderant advancement in the handling of these materials. From the point of view of electronic injection efficiency, however, insulating metal oxide matrices, as well as of most polymeric compounds so far employed, act as barriers for charges. So, efforts should focus on the search for novel semiconducting porous matrices or ways to improve the 


\section{WILEY-VCH}

charge transport properties of existing ones. In general, reducing the amount of host material relative to that of the active semiconductor will decrease the electrical resistance of the ensemble and hence improve its optoelectronic response. Also, the load of perovskite nanocrystals could be optimized to reach charge percolation through the nanocrystal sub-network. The risk, in this latter case, is coalescence of the nanocrystals, which would lead the ensemble to behave as a bulk material. Alternatively, methods for doping the matrix to change its transport properties might be explored. Overall, the use of porous matrices in which the void network determines or influences the physicochemical and optoelectronic properties of the guest perovskite compounds might open different routes for exploration of novel effects that can further enrich this already vigorous field.

\section{References}

[1] Z.-K. Tan, R. S. Moghaddam, M. L. Lai, P. Docampo, R. Higler, F. Deschler, M. Price, A. Sadhanala, L. M. Pazos, D. Credgington, F. Hanusch, T. Bein, H. J. Snaith, R. H. Friend, Nat. Nanotechnol. 2014, 9, 687.

[2] F. Deschler, M. Price, S. Pathak, L. E. Klintberg, D.-D. Jarausch, R. Higler, S. Hüttner, T. Leijtens, S. D. Stranks, H. J. Snaith, M. Atatüre, R. T. Phillips, R. H. Friend, J. Phys. Chem. Lett. 2014, 5, 1421.

[3] G. Xing, N. Mathews, S. S. Lim, N. Yantara, X. Liu, D. Sabba, M. Grätzel, S. Mhaisalkar, T. C. Sum, Nat. Mater. 2014, 13, 476.

[4] Y. Ling, Z. Yuan, Y. Tian, X. Wang, J. C. Wang, Y. Xin, K. Hanson, B. Ma, H. Gao, Adv. Mater. 2015, 28, 301.

[5] For a recent review on the subject please see M.E. Calvo, J. Mater. Chem. A 2017, 5, 20561 and references therein.

[6] J. P.Correa-Baena, M. Anaya, G. Lozano, W. Tress, K. Domanski, M. Saliba, T. Matsui, T.J. Jacobsson, M.E. Calvo, A. Abate, M. Graetzel, H. Miguez, A. Hagfeldt Adv. Mater. 2016, 28, 5031.

[7] M.D. Smith, H. I. Karunadasa, Acc. Chem. Res. 2018, 51, 619.

[8] G. Rainò, M.A. Becker, M.I. Bodnarchuk, R.F. Mahrt, M.V. Kovalenko, T. Stöferle, Nature 2018, 563, 671 . 


\section{WILEY-VCH}

[9] G. Li, Z.K. Tan, D. Di, M.L. Lai, L. Jiang, J.H.W. Lim, R.H. Friend, N.C. Greenham, NanoLett. 2015, 15, 2640.

[10] J. Byun, H. Cho, C. Wolf, M. Jang, A. Sadhanala, R.H. Friend, H. Yang, T-W. Lee, Adv. Mater. 2016, 28, 7515.

[11] N. Wang, L. Cheng, R. Ge, S. Zhang, Y. Miao, W. Zou, C. Yi, Y. Sun, Y. Cao, R. Yang, Y. Wei, Q. Guo, Y. Ke, M. Yu, Y. Jin, Y. Liu, Q. Ding, D. Di, L. Yang, G. Xing, H. Tian, C. Jin, F. Gao, R. H. Friend, J. Wang, W. Huang, Nature Photon. 2016, 10, 699.

[12] H. Huang, M. I Bodnarchuk, S. V. Kershaw, M. V. Kovalenko, A. L. Rogach, ACS Energy Lett. 2017, 2, 2071.

[13] S. Chang, Z. Bai, H. Zhong, Adv. Optical Mater. 2018, 6, 1800380.

[14] M.J. Grotevent, C.U. Hail, S. Yakunin, D.N. Dirin, K. Thodkar, G.B. Barin, P. GuyotSionnest, M. Calame, D. Poulikakos, M.V. Kovalenko, I. Shorubalko, Adv. Optical Mater. 2019, 1900019

[15] D. N. Dirin, L. Protesescu, D. Trummer, I. V. Kochetygov, S. Yakunin, F. Krumeich, N. P. Stadie, M. V. Kovalenko, Nano Lett. 2016, 9, 5866.

[16] V. Malgras, S. Tominaka, J. W. Ryan, J. Henzie, T. Takei, K. Ohara, Y. Yamauchi, J. Amer. Chem. Soc. 2016, 138, 13874.

[17] H.C. Wang, S.Y. Lin, A.C. Tang, B.P. Singh, H.C. Tong, C.Y. Chen, Y.C. Lee, T.L. Tsai, R.S. Liu, Angew. Chem. Int. Ed. 2016, 55, 7924.

[18] M. Anaya, A. Rubino, T.C. Rojas, J.F. Galisteo-López, M.E. Calvo, H. Míguez, Adv. Opt. Mater. 2017, 5, 1601087.

[19] L. C. Schmidt, A. Pertegas, S. González-Carrero, O. Malinkiewicz, S. Agouram, G. M. Espallargas, H. J. Bolink, R. E. Galian, J. Pérez-Prieto, J. Am. Chem. Soc. 2014, 136 , 850.

[20] S. T. Ha, X. Liu, Q. Zhang, D. Giovanni, T. C. Sum, Q. Xiong, Adv.Opt. Mater. 2014, 2 , 838.

[21] F. Zhang, H. Z. Zhong, C. Chen, X. G. Wu, X. M. Hu, H. L. Huang, J. B. Han, B. S. Zou, Y. P. Dong, ACS Nano 2015, 9 , 4533.

[22] L. Protesescu, S. Yakunin, M. I. Bodnarchuk, F. Krieg, R. Caputo, C. H. Hendon, R. X. Yang, A. Walsh, M. V. Kovalenko, Nano Lett. 2015, 15, 3692.

[23] M. Yuan, L.N. Quan, R. Comin, G. Walters, R. Sabatini, O. Voznyy, S. Hoogland, Y. Zhao, E.M. Beauregard, P. Kanjanaboos, Z. Lu, D.H. Kim, E.H. Sargent, Nature Nanotech. 2016, $11,872$.

[24] H. Huang, A. S. Susha, S. V. Kershaw, T. F. Hung, A. L. Rogach, Adv. Sci. 2015, 2, 1500194.

[25] S. Bai, Z. Yuan, F. Gao, J. Mater. Chem. C 2016, 4, 3898. 


\section{WILEY-VCH}

[26] Y. Hassan, Y. Song, R. D. Pensack, A. I. Abdelrahman, Y. Kobayashi, M. A. Winnik, G. D. Scholes, Adv. Mater. 2016, 28, 566 .

[27] J. C. Hamill, Jr., J. Schwartz,Y.-L. Loo, ACS Energy Lett. 2018, 3, 92-97

[28] Y. Wan, D. Y. Zhao Chem. Rev. 2007, 107, 2821.

[29] D. Grosso, F. Cagnol, G. J. de A. A. Soler-Illia, E. L. Crepaldi, P. A. Albouy, H. Amenitsch, A. Brunet-Bruneau and C. Sanchez, Adv. Funct. Mater. 2004, 14, 309.

[30] For the sake of clarity, we remind the reader that, in the classical $\mathrm{ABX}_{3}$ structure of metal halide based perovskites, $\mathrm{B}$ is a metal (mainly $\mathrm{Pb}$ or $\mathrm{Sn}$ ), $\mathrm{X}$ is a halide ( $\mathrm{I}, \mathrm{Br}$ or $\mathrm{Cl}$ ) and $\mathrm{A}$ is a monovalent cation that can be inorganic (Cs) or organic (such as alkylammonium or alkylamidinium).

[31] S. Demchyshyn, J.M.Roemer, H.Groi, H. Heilbrunner, C. Ulbricht, D. Apaydin, A. Böhm, U. Rütt, F. Bertram, G. Hesser, M. C. Scharber, N. S. Sariciftci, B. Nickel, S. Bauer, E. D. Głowacki, M. Kaltenbrunner, Sci. Adv. 2017, 3, 1700738

[32] R. B. Schoch, J. Han, P. Renaud, Rev. Mod. Phys. 2008, 80, 839

[33] W. Cha, H.J. Kim, S. Lee, J. Kim, J. Mater. Chem. C 2017, 5, 6667

[34] S.N. Raja, Y. Bekenstein, M.A. Koc, S. Fischer, D. Zhang, L. Lin, R.O. Ritchie, P. Yang, A.P. Alivisatos, ACS Appl. Mater. Interfaces 2016, 8, 35523

[35] Q. Zhou, Z. Bai, W.G. Lu, Y. Wang, B. Zou, H. Zhong, Adv. Mater. 2016, 28, 9163.

[36] Z. Li, L. Kong, S. Huang, L. Li, Angew. Chem. Int. Ed. 2017, 56, 8134

[37] T. Suteewong, H.Sai, R.Cohen, S. Wang, M. Bradbury, B. Baird, S. M. Gruner, U. Wiesner, J. Am. Chem. Soc. 2011,133, 2, 172-175

[38] P. Corell Escuina, A. García-Bennett, J. Vicente Ros-Lisa, A. Argüelles Foixa, A. Andrés, Food Chemistry 2017, 217, 360-363

[39] H.Miyata, S. Kobori, W. Kubo, M. Watanabe, K. Kuroda, Langmuir 2013, 29, 2, 761-765

[40] N. Hidalgo, C. Lopez-Lopez, G. Lozano, M. E. Calvo, H. Miguez, Langmuir 2012, 28, 39, 13777-13782.

[41] C. Pacholski, M. Sartor, M. J. Sailor, F. Cunin, G. M. Miskelly, J. Am. Chem. Soc. 2005, $127,33,11636-11645$

[42] D. Losic, M. Lillo, D. Losic Jr.,Small 2009,5, 12, 1392-1397

[43] V. Malgras, J. Henzie, T. Tankei, Y. Yamauchi, Chem. Commun. 2017, 53, 2359

[44] V. Malgras, J. Henzie, T. Takei, Y. Yamamuchi, Angew. Chem. Int. Ed. Int. Ed. 2018, 57, 1 [45] A.Rubino, M. Anaya, J.F. Galisteo-López, T.C. Rojas, M.E. Calvo, H. Míguez ACS Appl. Mater. Interfaces 2018, 10, 38334

[46] H.J. Lee, K.T. Cho, S. Paek, Y. Lee, A.J. Huckaba, V.I.E. Queloz, I. Zimmermann, G. Grancini, E. Oveisi, S. Yoo, S. Lee, T. Shin, M. Kim, M. K. Nazeeruddin, Adv. Funct. Mater. 2018, 28, 1803801 


\section{WILEY-VCH}

[47] J. Ghosh, R. Chosh, P.K. Giri, ACS Appl.Nano Mater. 2018, 1, 1551

[48] Q. Huo, D.I. Margolese, U. Ciesla, D.G. Demuth, P. Feng, T.E. Gier, P. Sieger, A. Firouzi, B.F. Chmelka, F. Schuth, G.D. Stucky, Chem. Mater. 1994, 6, 1176.

[49] D. Zhao, J. Feng, Q. Huo, N. Melosh, G. H. Fredrickson, B. F. Chmelka, G.D., Science 1998, 279, 548 .

[50] C.J. Bueno-Alejo, L.A. Villaescusa, A.E. Garcia-Bennett, Angew. Chemie-Int. Ed. 2014, $53,12106$.

[51] Z. Chen, Z.G. Gu, W. Q. Fu, F. Wang, J. Zhang, ACS Appl. Mater. Interfaces 2016, 8, 28737

[52] M. E. Calvo, O. Sánchez-Sobrado, S. Colodrero, H. Míguez, Langmuir, 2009, 25, 2443

[53] S. Ito, Y. Makari, T. Kitamura, Y. Wada, S. Yanagida, J. Mater. Chem. 2004, 14, 385

[54] F. Cebeci, Z. Wu, L. Zhai, R. E. Cohen, M. F. Rubner, Langmuir, 2006, 22, 2856

[55] S. Acharya, J.P. Hill, K. Ariga, Adv. Mater. 2009, 21, 2959

[56] M. Okuya, K. Nakade. S. Kaneko, Sol. Energy Mater. Sol. Cells 2002, 70, 425.

[57] S. Parveen, K. K. Paul, R. Das, P.K. Giri J. Colloid Interface Sci. 2019, 539, 619

[58] D. Takhellambam, T. R. Meena, D. Jana, Chem. Commun. 2019, 55, 4785

[59 ] V. Malgras, A. Nattestad, J. H. Kim, S. X. Dou, Y. Yamauchi, Sci. Technol. Adv. Mater. 2017, 1, 334 .

[60] D. Di, K. P. Musselman, G. Li, A. Sadhanala, Y. Ievskaya, Q. Song, Z. Tan, M. Ling Lai, J. L. MacManus-Driscoll, N. C. Greenham, R. H. Friend, J. Phys. Chem. Lett. 2015, 6, 446

[61] L. E. Brus, J. Chem. Phys. 1984, 80, 4403.

[62] Al. L. Éfros, A. L. Éfros, Sov. Phys. Semicond. 1982, 16, 1209.

[63] V. D’Innocenzo, G. Grancini, M. J. P. Alcocer, A. R. S. Kandada, S. D. Stranks, M. M. Lee, G. Lanzani, H. J. Snaith, A. Petrozza, Nat. Commun. 2014, 5, 3585

[64] K. Tanaka, T, Takahashi, T, Ban, T, Kondo, K, Uchida, N, Miura, Solid State Communications, 2003, 127, 619-623

[65] L. Protesescu, S. Yakunin, M. I. Bodnarchuk, F. Krieg, R. Caputo, C. H. Hendon, R. X. Yang, A. Walsh, and Maksym V. Kovalenko, Nano Lett. 2015, 15, 3692-3696

[66] N. Aristidou, I. Sanchez-Molina, T. Chotchuangchutchaval, M. Brown, L. Martinez, T. Rath, S. A. Haque, Angew. Chem. 2015, 127, $8326-8330$

[67] M.Anaya, J. F. Galisteo-Lopez, M. E. Calvo, J. P. Espinos, H. Míguez, J. Phys. Chem. Lett. 2018, 9, 3891-3896

[68] J. Chun-Ren Ke, A. S. Walton, D. J. Lewis, A. Tedstone, P. O’Brien, A. G. Thomas, W. R. Flavell, Chem. Commun., 2017, 53, 5231

[69] Y.H. Kye, C.J. Yu, U.G. Jong, Y. Chen, A. Walsh, J. Phys. Chem. Lett. 2018, 9, 2196-2201 


\section{WILEY-VCH}

[70] Y. Wang, J. He, H. Chen, J. Chen, R. Zhu, P. Ma, A.Towers, Y. Lin, A. J. Gesquiere, S.Wu, Y. Dong Adv.Mater. 2016, 28, 10710

[71] Su, Y.; Chen, X.; Ji, W.; Zeng, Q.; Ren, Z.; Su, Z.; Liu, L. ACS Appl. Mater. Interfaces 2017, 9, 33020

[72] F.J. Galisteo-López, M. Anaya, M. E. Calvo, H. Miguez, J. Phys. Chem. Lett., 2015, 6 2200-2205

[73] Z. Andaji-Garmaroudi, M. Anaya, A. J. Pearson, S. D. Stranks, Adv. Energy Mater. 2019, 1903109

[74] M. Gerhard, B. Louis, R. Camacho, A. Merdasa, J. Li, A. Kiligaridis, A. Dobrovolsky, J. Hofkens, I.G. Scheblykin, Nat. Comm., 2019,10,1698

[75] Y.-H. Kim, C. Wolf, H. Kim, T.-W. Lee, Nano Energy, 2018, 52, 329.

[76] F. Di Stasio, S. Christodoulou, N. Huo, G. Konstantatos, Chem. Mater. 2017, 29, 7663

[77] X. Gong, O. Voznyy, A. Jain, W. Liu, R. Sabatini, Z. Piontkowski, G. Walters, G. Bappi, S. Nokhrin, O. Bushuyev, M. Yuan, R. Comin, D. McCamant, S.O. Kelley, E.H. Sargent, Nat. Mater. 2018, 17, 550.

[78] J. Kang, L .W. Wang, J. Phys. Chem. Lett. 2017, 8, 489-493

[79] S. ten Brinck, F. Zaccaria, I. Infante, ACS Energy Lett. 2019, 4, 2739-2747

[80] R. Godin, X. Ma, S. González-Carrero, T. Du, X. Li, C. T. Lin, M. A. McLachlan, R. E. Galian, J. Pérez-Prieto, J. R. Durrant, Adv. Opt. Mater. 2018, 6, 1701203

[81] S.M. Yoo, S. Joon Yoon, J. A. Anta, H. Joong Lee, P. P. Boix, I. Mora-Sero, Joule, 2019, $3,1-15$

[82] A. Vassilakopoulou, D. Papadatos, I. Koutselas, Micropor. Mesopor. Mat., 2017, 249, $165-$ 175 


\section{WILEY-VCH}

\section{Acknowledgements}

The research leading to these results has received funding from the Spanish Ministry of Economy and Competitiveness under grant MAT2017-88584-R (AEI/FEDER,UE). This work was devised and partly realized while H.M. was a Macquarie University Visiting Fellow. 


\section{WILEY-VCH}

Synthesis of metal halide perovskites within porous matrices allows achieving a fine control over the semiconductor crystal size at the nanoscale. These methods arise as a promising alternative to those based on colloidal nucleation and growth as they permit attaining nanocrystal loaded films of optical quality, without using organic ligands, with improved stability and in a configuration suitable for applications in optoelectronics.

Keywords: perovskite nanocrystals, porous materials, scaffold controlled synthesis, optoelectronic properties.

\section{Mesoporous matrices as hosts for perovskite nanocrystals}

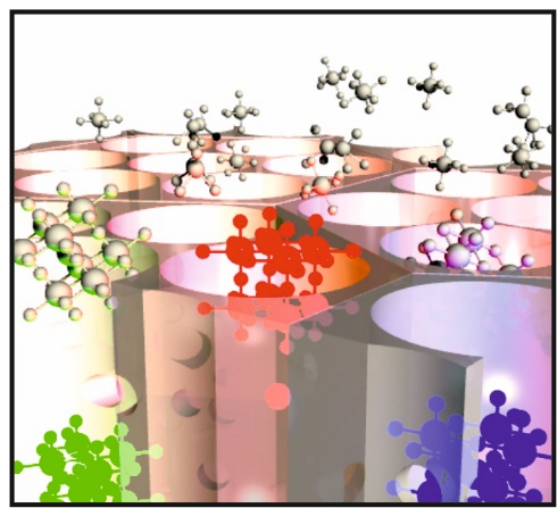

Received: ((will be filled in by the editorial staff))

Revised: ((will be filled in by the editorial staff))

Published online: ((will be filled in by the editorial staff)) 